\title{
RECUPERACIÓN DEMOGRÁFICA EN LOS MUNICIPIOS RURALES DE LA «MONTAÑA DE ALICANTE». NUEVAS TENDENCIAS EN UNA POBLACIÓN FUERTEMENTE ENVEJECIDA
}

\author{
Carlos Cortés Samper \\ Departamento de Geografía Humana \\ Universidad de Alicante
}

\section{RESUMEN}

El éxodo rural fue el factor clave para entender el comportamiento demográfico de los municipios rurales de la «Montaña de Alicante» durante gran parte del siglo XX. Sin embargo, según los últimos recuentos de población se constata la recuperación demográfica positiva en la mayor parte de estos municipios, aunque en algunos, todavía hoy, continúan produciéndose pérdidas de población. Este doble comportamiento es significativo de los nuevos procesos que se dan actualmente en el medio rural. La revalorización de los espacios rurales ha contribuido a que aparezcan en ellos nuevas funciones, y que se consideren como idóneos para residir por la consideración de lugares tranquilos y próximos a la naturaleza, en clara oposición a los medios urbanos. Los saldos migratorios positivos son la causa de esa recuperación, pero este hecho no debe enmascarar otros problemas demográficos que aún perduran, como son los saldos naturales negativos, el fuerte envejecimiento y las altas tasas de dependencia. La recuperación demográfica es clave para entender el futuro del conjunto de estos municipios.

Palabras clave: Despoblamiento, éxodo rural, municipios rurales, recuperación demográfica, provincia de Alicante.

\begin{abstract}
For most of the twentieth century, rural exodus played the key role in demographic tendencies in the rural towns of the «Montaña de Alicante». However, according to recent population counts, most of these towns are now experiencing a demographic recovery, although some areas are still in the midst of a rural exodus. This dual behaviour reveals the new processes currently occurring in rural settings. The revaluing of rural areas has contributed to the appearance of new functions there and to the fact that they are now considered to be ideal living spaces since they are peaceful and close to nature, in clear contrast to urban areas. This recovery is due to the phenomenon of in-migration, although
\end{abstract}


this should not conceal other more persistent demographic problems, such as the natural flow of out-migration, the large ageing population and high rates of dependency. Demographic recovery is a key factor in understanding the future of these towns as a whole.

Keywords: Depopulation, rural exodus, rural towns, demographic recovery, province of Alicante.

\section{Introducción}

La zona objeto de estudio está formada por 42 municipios (mapa 1) que pertenecen a 5 comarcas distintas de la provincia de Alicante: l'Alcoià, el Comtat, la Marina Alta, la Marina Baixa y l'Alacantí. Este ámbito geográfico se caracteriza, desde un punto de vista físico, por la alternancia de valles y de montañas, constituyendo un área que puede ser considerada como un todo, donde la ruralidad de sus municipios es el principal factor a tener en cuenta. Se han considerado los municipios de menos de 2.000 habitantes ${ }^{1}$, excluyendo a las cabeceras comarcales que por su mayor dinamismo tienen comportamientos diferenciados respecto a los municipios propiamente rurales.

Los límites de este ámbito no están muy definidos, pues no todos los municipios tienen las mismas características geográficas y la delimitación en las zonas de borde, considerando las semejanzas, queda un tanto difuminada.

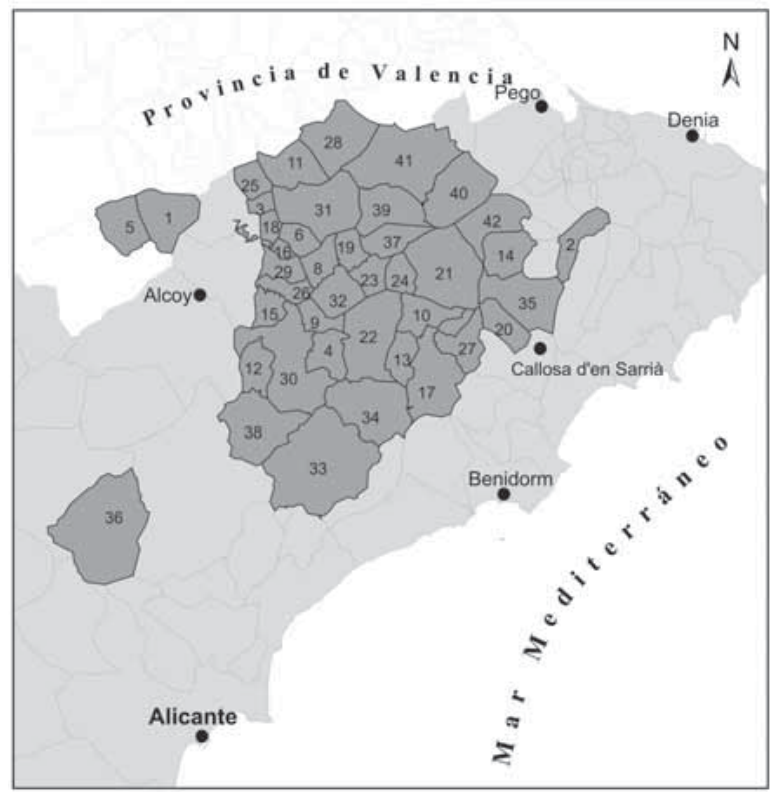

Fuente: Elaboración propia

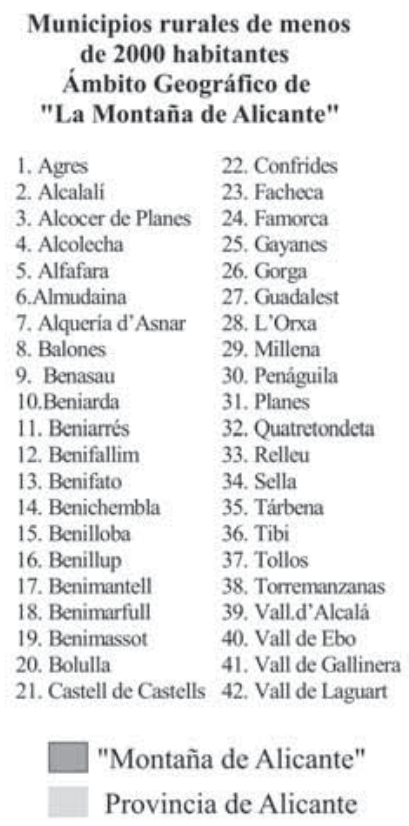

Mapa 1. Ámbito geográfico de los municipios rurales de menos de 2.000 habitantes de la «Montaña de Alicante».

1 Este es el límite de población utilizado por el INE para definir un municipio como rural. 


\section{El proceso de éxodo rural durante el siglo $\mathrm{XX}$}

Durante el siglo XX el éxodo rural ha sido el proceso que ha caracterizado el comportamiento demográfico de este conjunto de municipios. Las causas para entender este proceso se deben, sobre todo, a la crisis agraria general y la consecuente búsqueda de alternativas, causa del intenso despoblamiento (GOZÁLVEZ, 1972: 43). En el ámbito de la Montaña de Alicante destaca una doble influencia sobre su emigración, por un lado la ejercida por la actividad industrial textil desarrollada en Alcoy y que, por sus necesidades de mano de obra durante la primera mitad del siglo XX, determinó la creación de flujos migratorios desde los municipios rurales cercanos (PÉREZ, 1997: 51). Por otro, el desarrollo económico turístico más tardío del litoral de la provincia de Alicante, también supuso la creación de flujos emigratorios desde los municipios del interior próximos a las franjas litorales debido a la oferta de empleos generados por la explotación turística.

Tomando los datos de forma conjunta, es decir para el total de municipios analizados, se aprecia mejor la enorme pérdida de población que sufrieron estos municipios rurales durante todo el siglo XX (tabla 1 y gráfico 1).

Tabla 1

EVOLUCIÓN DE LA POBLACIÓN CENSAL DESDE 1900 A 2001. ÁMBITO GEOGRÁFICO DE LA «MONTAÑA DE ALICANTE»

\begin{tabular}{|c|c|c|c|c|c|c|c|c|c|c|}
\hline $\mathbf{1 9 0 0}$ & $\mathbf{1 9 1 0}$ & $\mathbf{1 9 2 0}$ & $\mathbf{1 9 3 0}$ & $\mathbf{1 9 4 0}$ & $\mathbf{1 9 5 0}$ & $\mathbf{1 9 6 0}$ & $\mathbf{1 9 7 0}$ & $\mathbf{1 9 8 1}$ & $\mathbf{1 9 9 1}$ & $\mathbf{2 0 0 1}$ \\
\hline 39.099 & 37.470 & 36.130 & 34.879 & 33.814 & 32.335 & 28.134 & 24.093 & 20.226 & 18.126 & 18.089 \\
\hline 100 & 95,8 & 92,4 & 89,2 & 86,5 & 82,7 & 71,9 & 61,6 & 51,7 & 46,3 & 46,2 \\
\hline
\end{tabular}

Fuente: INE. Elaboración propia.

Tanto en la tabla 1 como en el gráfico 1 , se observa la tendencia negativa seguida durante el pasado siglo por la población del conjunto de municipios, sobre todo entre 1950 y 1981; sin embargo en la década de los noventa se ha producido una estabilización de las cifras censales con tan sólo 37 pérdidas, proceso que contrasta con la situación de los intercensales anteriores.

Para completar el análisis de la evolución demográfica, en la tabla 2 se incluyen tanto las variaciones absolutas como relativas para los periodos censales del pasado siglo. Aunque el despoblamiento es continuado en el área rural estudiada, se puede hablar de tres fases en cuanto al comportamiento demográfico en el ámbito de la «Montaña de Alicante»:

- Una primera fase comprende desde 1900 hasta 1950, donde las pérdidas son continuas, en torno al $-4 \%$ para cada uno de los intercensales.

- La segunda abarca el periodo 1950-1991, en el que se produce una pérdida acentuada de habitantes, con valores superiores a $-10 \%$ en períodos decenales; son muy significativas las pérdidas durante los intercensales 1960-1970 y 1971-1981, cuando se pierde hasta un -14 y un $-16 \%$ de la población de esta área rural.

- La tercera fase queda limitada al último intercensal, cuando se produce un destacado freno en las pérdidas. Así durante el periodo 1991-2001 tan sólo se ha producido una variación del $-0,2 \%$. 


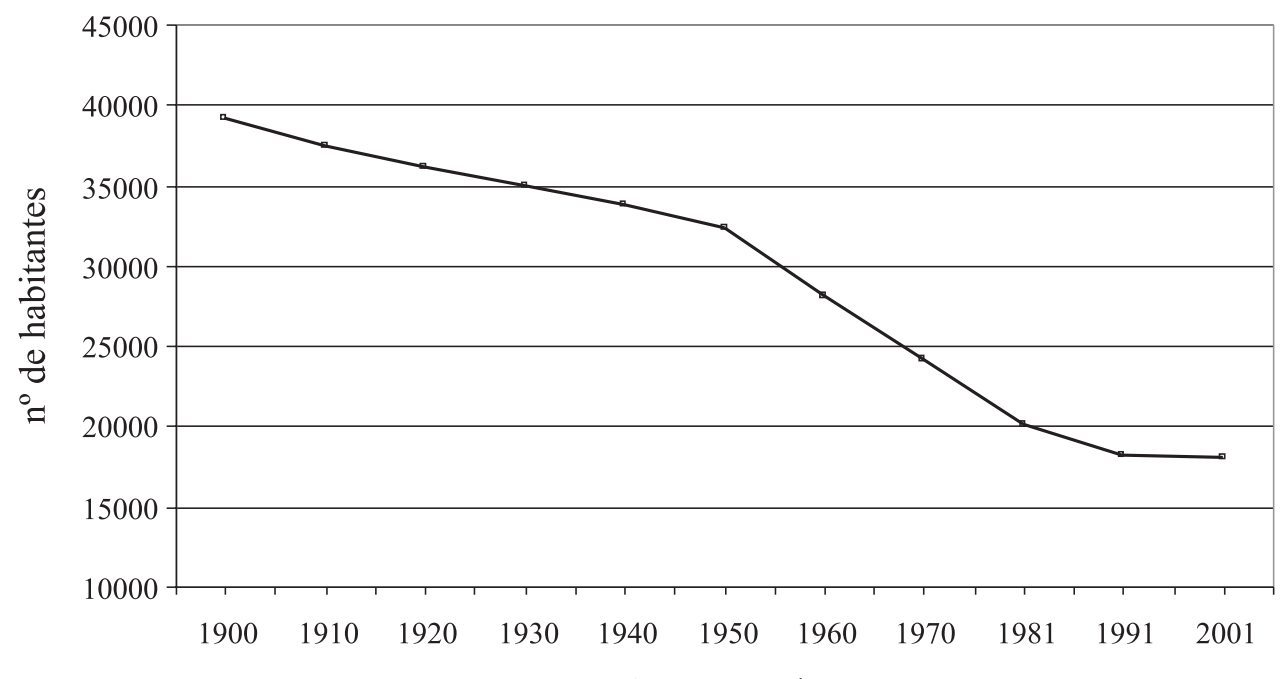

Años censales

GrÁFICO 1. Evolución de la población durante el siglo XX. Municipios rurales de la montaña de Alicante.

Fuente: INE. Elaboración propia.

Tabla 2

VARIACIONES DECENALES DE POBLACIÓN DEL CONJUNTO DE MUNICIPIOS RURALES ANALIZADOS. CIFRAS ABSOLUTAS Y RELATIVAS

\begin{tabular}{|c|c|c|}
\hline Periodos intercensales & $\begin{array}{c}\text { Variación absoluta de } \\
\text { población }\end{array}$ & $\begin{array}{c}\text { Variación relativa de la } \\
\text { población (\%) }\end{array}$ \\
\hline $1900-1910$ & -1.629 & $-4,1$ \\
\hline $1910-1920$ & -1.340 & $-3,6$ \\
\hline $1920-1930$ & -1.251 & $-3,5$ \\
\hline $1930-1940$ & -1.092 & $-3,1$ \\
\hline $1940-1950$ & -1.492 & $-4,2$ \\
\hline $1950-1960$ & -4.201 & $-12,99$ \\
\hline $1960-1970$ & -4.041 & $-14,3$ \\
\hline $1970-1981$ & -3.867 & $-16,05$ \\
\hline $1981-1991$ & -2.100 & $-10,38$ \\
\hline $\mathbf{1 9 9 1 - 2 0 0 1}$ & $\mathbf{- 3 7}$ & $\mathbf{- 0 , 2 0}$ \\
\hline
\end{tabular}

Fuente: INE. Elaboración propia.

La variación durante la última década, si bien continúa siendo negativa, ha supuesto un cambio de tendencia respecto a las pérdidas de los intercensales anteriores y supone el punto de partida para analizar las causas y las nuevas dinámicas que se están produciendo 
en la actualidad. La situación de crisis demográfica (MATARREDONA, 1996: 41-60), parece que deja paso a un estancamiento de las pérdidas, aunque este es reflejo de un doble comportamiento, en el que unos municipios recuperan población y otros continúan con pérdidas, pero menos significativas que en épocas anteriores.

\section{La evolución reciente de la población}

En la tabla 3 se indican las variaciones demográficas para los últimos tres intercensales, 1970-1981, 1981-1991 y 1991-2001.

La comparación de los tres periodos censales analizados demuestra cómo se ha producido un cambio de tendencia demográfica durante el último decenio. Si en el primer intercensal (1970-1981) 41 municipios perdían población, ya en el siguiente periodo (1981-1991) éstos se redujeron a 35 municipios, con 6 casos de aumento demográfico. Pero será entre los años 1991 y 2001 cuando se ha producido un claro cambio de tendencia, con 19 municipios que han continuado perdiendo población mientras otros 22 han tenido incrementos demográficos respecto a las cifras de 1991 .

Por tanto, el cambio de tendencia ya se intuye durante la década de los ochenta (se pasa de un decrecimiento de $-16 \%$ en la década de los setenta, a un $-10 \%$ en la de los ochenta). La constatación de esta tendencia durante el decenio posterior, con una variación negativa de tan sólo $-0,2 \%$. Aunque este proceso no está generalizado en todos los municipios, sino que aparecen comportamientos opuestos en los municipios del ámbito geográfico de estudio.

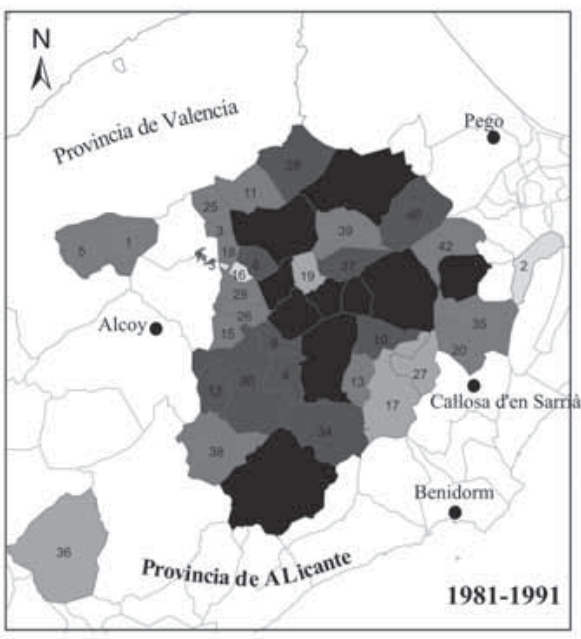

$$
\begin{aligned}
& \text { Intervalos de variación } \\
& \text { de población (en \%) } \\
& <(-20) \\
& >(-20)<(-10,0) \\
& \square(-10)<(-0,0) \\
& \square>0<10 \\
& \square>10,0
\end{aligned}
$$
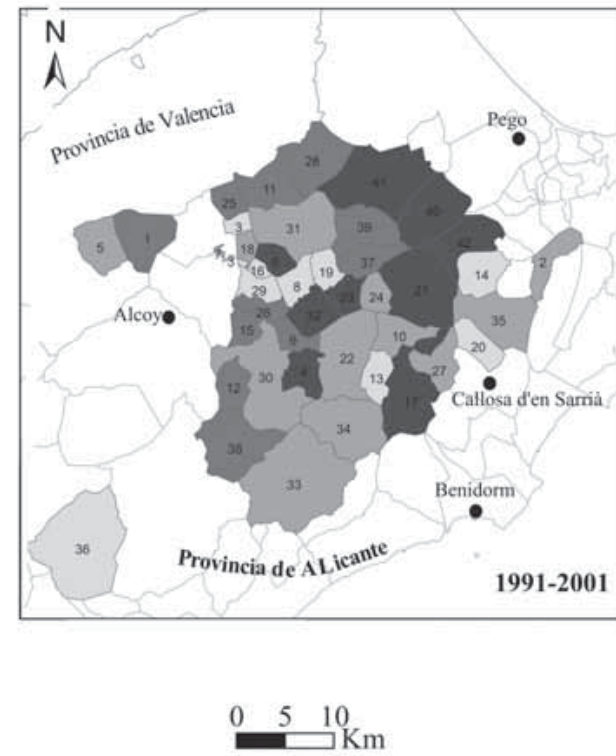

Fuente: Censos de población de España. Elaboración propia

MAPA 2. Variaciones de población en los periodos censales 1981-1991 y 1991-2001. 
Tabla 3

VARIACIONES ABSOLUTAS Y RELATIVAS DE LA POBLACIÓN DURANTE LOS TRES ÚLTIMOS INTERCENSALES EN LOS MUNICIPIOS RURALES LA «MONTAÑA DE ALICANTE». PERIODOS 1970-81, 1981-91 y 1991-2001

\begin{tabular}{|c|c|c|c|c|c|c|c|c|c|}
\hline \multirow[b]{2}{*}{ Municipios } & \multicolumn{3}{|c|}{ Variación 1970-1981 } & \multicolumn{3}{|c|}{ Variación 1981-1991 } & \multicolumn{3}{|c|}{ Variación 1991-2001 } \\
\hline & Pob. 1981 & v.a. & $\%$ & Pob. 1991 & v.a. & $\%$ & Pob. 2001 & v.a. & $\%$ \\
\hline Agres & 727 & -162 & $-18,2$ & 676 & -51 & $-7,02$ & 635 & -41 & $-6,07$ \\
\hline Alcalalí & 624 & -65 & $-9,4$ & 827 & 203 & 32,53 & 902 & 75 & 9,07 \\
\hline Alcocer de Planes & 132 & -52 & $-28,3$ & 123 & -9 & $-6,82$ & 153 & 30 & 24,39 \\
\hline Alcoleja & 346 & -99 & $-22,2$ & 297 & -49 & $-14,16$ & 251 & -46 & $-15,49$ \\
\hline Alfafara & 404 & -58 & $-12,6$ & 399 & -5 & $-1,24$ & 418 & 19 & 4,76 \\
\hline Almudaina & 162 & -66 & $-28,9$ & 133 & -29 & $-17,90$ & 109 & -24 & $-18,05$ \\
\hline L'Alqueria d'Asnar & 412 & -137 & $-25,0$ & 408 & -4 & $-0,97$ & 417 & 9 & 2,21 \\
\hline Balones & 204 & -47 & $-18,7$ & 152 & -52 & $-25,49$ & 185 & 33 & 21,71 \\
\hline Benasau & 254 & -90 & $-26,2$ & 221 & -33 & $-12,99$ & 221 & 0 & 0,00 \\
\hline Beniardà & 277 & -49 & $-15,0$ & 231 & -46 & $-16,60$ & 245 & 14 & 6,06 \\
\hline Beniarrés & 1.633 & -102 & $-5,9$ & 1.503 & -130 & $-7,96$ & 1375 & -128 & $-8,52$ \\
\hline Benifallim & 196 & 12 & 6,5 & 166 & -30 & $-15,31$ & 155 & -11 & $-6,63$ \\
\hline Benifato & 124 & -42 & $-25,3$ & 124 & 0 & 0,00 & 166 & 42 & 33,87 \\
\hline Benigembla & 482 & -62 & $-11,4$ & 354 & -128 & $-26,56$ & 488 & 134 & 37,85 \\
\hline Benilloba & 1.036 & -161 & $-13,5$ & 959 & -77 & $-7,43$ & 903 & -56 & $-5,84$ \\
\hline Benillup & 62 & -27 & $-30,3$ & 76 & 14 & 22,58 & 97 & 21 & 27,63 \\
\hline Benimantell & 425 & -66 & $-13,4$ & 453 & 28 & 6,59 & 402 & -51 & $-11,26$ \\
\hline Benimarfull & 420 & -112 & $-21,1$ & 416 & -4 & $-0,95$ & 420 & 4 & 0,96 \\
\hline Benimassot & 115 & -42 & $-26,8$ & 118 & 3 & 2,61 & 147 & 29 & 24,58 \\
\hline Bolulla & 306 & -150 & $-32,9$ & 277 & -29 & $-9,48$ & 358 & 81 & 29,24 \\
\hline Castell de Castells & 695 & -102 & $-12,8$ & 532 & -163 & $-23,45$ & 471 & $\begin{array}{c}01 \\
-61 \\
\end{array}$ & $-11,47$ \\
\hline Confrides & 377 & -139 & $-26,9$ & 297 & -80 & $-21,22$ & 309 & 12 & 4,04 \\
\hline Facheca & 178 & -55 & $-23,6$ & 137 & -41 & $-23,03$ & 115 & -22 & $-16,06$ \\
\hline Famorca & 126 & -44 & $-25,9$ & 70 & -56 & $-44,44$ & 73 & 3 & 4,29 \\
\hline Gaianes & 358 & -82 & $-18,6$ & 331 & -27 & $-7,54$ & 319 & -12 & $-3,63$ \\
\hline Gorga & 323 & -43 & $-11,7$ & 291 & -32 & $-9,91$ & 269 & -22 & $-7,56$ \\
\hline Guadalest & 164 & -57 & $-25,8$ & 165 & 1 & 0,61 & 180 & 15 & 9,09 \\
\hline L'Orxa & 1.002 & -50 & $-4,8$ & 810 & -192 & $-19,16$ & 737 & -73 & $-9,01$ \\
\hline Millena & 140 & -28 & $-16,7$ & 135 & -5 & $-3,57$ & 178 & 43 & 31,85 \\
\hline Penàguila & 423 & -188 & $-30,8$ & 352 & -71 & $-16,78$ & 357 & 5 & 1,42 \\
\hline Planes & 968 & -119 & $-10,9$ & 769 & -199 & $-20,56$ & 787 & 18 & 2,34 \\
\hline Quatretondeta & 266 & -26 & $-8,9$ & 200 & -66 & $-24,80$ & 166 & -34 & $-17,00$ \\
\hline Relleu & 1.012 & -308 & $-23,3$ & 742 & -270 & $-26,68$ & 800 & 58 & 7,82 \\
\hline Sella & 690 & -223 & $-24,4$ & 590 & -100 & $-14,49$ & 591 & 1 & 0,17 \\
\hline Tàrbena & 723 & -331 & $-31,4$ & 713 & -10 & $-1,38$ & 715 & 2 & 0,28 \\
\hline Tibi & 986 & -57 & $-5,5$ & 1.045 & 59 & 5,98 & 1252 & 207 & 19,81 \\
\hline Tollos & 49 & -64 & $-56,6$ & 42 & -7 & $-14,29$ & 41 & -1 & $-2,38$ \\
\hline Torremanzanas & 782 & -114 & $-12,7$ & 736 & -46 & \begin{tabular}{|l|}
$-5,88$ \\
\end{tabular} & $\begin{array}{r}71 \\
704\end{array}$ & \begin{tabular}{|c|}
-32 \\
-32
\end{tabular} & $-4,35$ \\
\hline Vall d'Alcalà & 180 & -133 & $-42,5$ & 174 & -6 & $-3,33$ & 166 & -8 & $-4,60$ \\
\hline Vall de Ebo & 431 & -30 & $-6,5$ & 359 & -72 & $-16,70$ & 318 & -41 & $-11,42$ \\
\hline Vall de Gallinera & 930 & -178 & $-16,1$ & 735 & -195 & $-20,97$ & 624 & -111 & $-15,10$ \\
\hline Vall de Laguar & 1.082 & -178 & $-14,1$ & 988 & -94 & $-8,69$ & 870 & -118 & $-11,94$ \\
\hline Total & 20.226 & -4126 & $-16,9$ & 18.126 & -2100 & $-10,38$ & 18.089 & -37 & $-0,20$ \\
\hline
\end{tabular}

Fuente: INE. Elaboración propia. 
En este doble comportamiento no todos los municipios registran llegadas de nuevos residentes - causa principal de la recuperación demográfica - , y además los saldos naturales negativos contribuyen a mantener las pérdidas demográficas, al ser muy superiores a los flujos de llegadas.

En el mapa adjunto se pueden observar las diferencias en cuanto al cambio de tendencia ocurrido durante los últimos dos periodos censales. La trama utilizada permite diferenciar cómo los municipios afectados por la pérdida demográfica (tonos oscuros) se han reducido entre los dos periodos considerados, generalizándose los municipios que ganan población (tonos claros)

\section{Causas del cambio de tendencia}

Atendiendo a los saldos naturales y migratorios de las últimas décadas (ver tabla 4), se demuestra que la recuperación demográfica se ha dado, principalmente, por los saldos migratorios positivos durante los últimos diez años, a pesar de que los saldos naturales han continuado siendo negativos por el elevado grado de envejecimiento. Los flujos de llegada llegan a contrarrestar las pérdidas originadas por los saldos naturales.

Tabla 4

TASA ANUAL MEDIA DE VARIACIÓN ACUMULADA PARA LOS MUNICIPIOS RURALES DE LA «MONTAÑA DE ALICANTE». PERIODOS 1981-1991 Y 1991-2001.

\begin{tabular}{|c|c|c|c|c|c|}
\hline & Pob. inicial & Pob. final & $\begin{array}{c}\text { C.V (saldo } \\
\text { natural) }\end{array}$ & $\begin{array}{c}\text { Saldo } \\
\text { migratorio }\end{array}$ & $\begin{array}{c}\text { r \% } \\
\text { intercensal }\end{array}$ \\
\hline $1981-1991$ & 20.226 & 18.126 & -1.202 & -898 & $\mathbf{- 1 , 0 9 0 2}$ \\
\hline $1991-2001$ & 18.126 & 18.089 & -1.702 & 1.665 & $\mathbf{- 0 , 0 2 2 7}$ \\
\hline
\end{tabular}

Fuente: INE e IVE. Censo de Población 1981, 1991 y 2001.

Como se observa en la tabla, la consideración general del conjunto de los municipios analizados determina valores de tasa anual media de variación ${ }^{2}$ negativos (expresada como $\mathrm{r} \%$ intercensal en la tabla) para los dos periodos de tiempo analizados, aunque la tendencia ha sido a disminuir esa tasa negativa desde el $-1,09 \%$, alcanzada durante la década de los ochenta, hasta el $-0,02 \%$ del último decenio. Es decir, se ha producido una reducción del decrecimiento demográfico que puede ser considerada como importante

Analizando tanto los saldos naturales como los migratorios, podemos hacernos una idea de cuales han sido las causas que explican el cambio de tendencia.

Anteriormente se ha comentado que los incrementos de población se deben, en el caso analizado, sobre todo a la influencia de los saldos migratorios y no por crecimientos relacionados con los saldos naturales. Estos crecimientos se producen porque se contrarrestan las fuertes pérdidas debidas al saldo natural con los nuevos flujos inmigratorios. En otros municipios, en cambio, a pesar de los saldos migratorios positivos, las pérdidas demográficas por saldos naturales negativos son, aún, demasiado elevadas. Véase la tabla 5, donde se muestran estos comportamientos:

2 El cálculo de r, o tasa anual media de variación acumulada, se calcula a partir de la fórmula:

$\mathrm{r}=\left(\mathrm{P}^{\mathrm{t}+\mathrm{a}} / \mathrm{P}^{\mathrm{t}}\right)^{1 / \mathrm{a}}-1$, utilizada por J.Vinuesa Angulo (editor) y otros. (1997): Demografía, análisis y proyecciones, Ed. Síntesis, Madrid, 366 páginas. 
Tabla 5

SALDOS NATURALES Y MIGRATORIOS, CRECIMIENTO INTERCENSAL Y TASA DE CRECIMIENTO REAL DE LOS MUNICIPIOS RURALES DE LA «MONTAÑA DE ALICANTE». PERIODO 1991-2001

\begin{tabular}{|c|c|c|c|c|}
\hline Municipios & $\begin{array}{l}\text { Crecimiento } \\
\text { Intercensal }\end{array}$ & Saldo Natural & $\begin{array}{c}\text { Saldo } \\
\text { migratorio }\end{array}$ & $\begin{array}{c}\text { Tasa de } \\
\text { crecimiento } \\
\text { real }(\%)\end{array}$ \\
\hline Agres & -41 & -42 & 1 & $-5,70$ \\
\hline Alcalalí & 75 & -83 & 158 & 7,89 \\
\hline Alcocer de Planes & 30 & -8 & 38 & 19,76 \\
\hline Alcolecha & -46 & -49 & 3 & $-15,26$ \\
\hline Alfafara & 19 & 20 & -1 & 4,23 \\
\hline Almudaina & -24 & -7 & -17 & $-18,00$ \\
\hline L'Alqueria d'Asnar & 9 & 2 & 7 & 1,98 \\
\hline Balones & 33 & -9 & 42 & $\mathbf{1 7 , 8 0}$ \\
\hline Benasau & 0 & -38 & 38 & $\mathbf{0 , 0 0}$ \\
\hline Beniardà & 14 & -16 & 30 & 5,35 \\
\hline Beniarrés & -128 & -57 & -71 & $-8,09$ \\
\hline Benigombla & 134 & -55 & 189 & 28,94 \\
\hline Benifallim & -11 & -19 & 8 & $-6,23$ \\
\hline Benifato & 42 & -11 & 53 & 26,33 \\
\hline Benilloba & -56 & -70 & 14 & $-6,47$ \\
\hline Benillup & 21 & -11 & 32 & 22,07 \\
\hline Benimantell & -51 & -35 & -16 & $-10,85$ \\
\hline Benimarfull & 4 & -56 & 60 & $\mathbf{0 , 8 7}$ \\
\hline Benimasot & 29 & -11 & 40 & 19,90 \\
\hline Bolulla & 81 & -34 & 115 & 23,19 \\
\hline Castell de Castells & -61 & -97 & 36 & $-11,00$ \\
\hline Confrides & 12 & -36 & 48 & 3,60 \\
\hline Cuatretondeta & -34 & -32 & -2 & $-16,90$ \\
\hline Facheca & -22 & -17 & -5 & $-15,87$ \\
\hline Famorca & 3 & -9 & 12 & $\mathbf{3 , 8 1}$ \\
\hline Gaianes & -12 & -32 & 20 & $-3,36$ \\
\hline Gorga & -22 & -23 & 1 & $-7,14$ \\
\hline Guadalest & 15 & -23 & 38 & 7,91 \\
\hline L'Orxa & -73 & -54 & -19 & $-8,58$ \\
\hline Millena & 43 & -22 & 65 & 24,98 \\
\hline Penàguila & 5 & -28 & 33 & 1,28 \\
\hline Planes & 18 & -82 & 100 & 2,10 \\
\hline Relleu & 58 & -110 & 168 & 6,84 \\
\hline Sella & 1 & -72 & 73 & $\mathbf{0 , 1 5}$ \\
\hline Tàrbena & 2 & -72 & 74 & 0,25 \\
\hline Tibi & 207 & 3 & 204 & 16,39 \\
\hline Tollos & -1 & 1 & -2 & $-2,20$ \\
\hline Torremanzanas & -32 & -71 & 39 & $-4,04$ \\
\hline Vall d'Alcalà & -8 & -27 & 19 & $-4,28$ \\
\hline Vall de Ebo & -41 & -59 & 18 & $-11,01$ \\
\hline Vall de Gallinera & -111 & -86 & -25 & $-14,85$ \\
\hline Vall de Laguart & -118 & -165 & 47 & $-11,50$ \\
\hline Total & -37 & -1.702 & 1.665 & $-0,19$ \\
\hline
\end{tabular}

Fuente: INE, Censos de población; IVE, Anuaris Estadistics de la Comunitat Valenciana. Elaboración propia. 
En cuanto a las cifras globales, puede observarse cómo el saldo migratorio es claramente positivo (1.665 nuevos habitantes), mientras que el crecimiento natural continua siendo negativo (-1.702).

Durante la década de los noventa 19 de los municipios analizados tuvieron incrementos demográficos originados por los altos saldos migratorios que superaron a los saldos naturales negativos, estos son: Alcalalí, Alcocer de Planes, Balones, Beniardà, Benichembla, Benifato, Benillup, Benimarfull, Benimassot, Bolulla, Confrides, Famorca, Guadalest, Millena, Penàguila, Planes, Relleu, Sella y Tàrbena. En otros 11, a pesar de los saldos migratorios positivos, estos no alcanzan a contrarrestar los saldos naturales negativos; pero denotan que a pesar de los efectos del envejecimiento de la población y la consecuente alta tasa de mortalidad, los flujos de nuevos residentes pueden constituir un elemento clave para la recuperación sociodemográfica. La excepción es el municipio de Benasau que no ha variado su población en el periodo 1991-2001, con saldos naturales y migratorios que se contrarrestan.

Además, existen otros cuatro casos con comportamientos que pueden ser clasificados como peculiares. Así, Alfafara y Tollos tienen saldos naturales positivos y saldos migratorios negativos. La explicación, para el primero de ellos, puede estar en la existencia de una importante población joven que permite que la natalidad sea bastante alta, mientras que se producen flujos emigratorios también de la población joven, posiblemente por la falta de oportunidades; en Tollos su reducido número de habitantes determina que se produzcan variaciones mínimas en los saldos naturales y migratorios. El segundo comportamiento peculiar es el de los municipios de Alqueria d'Asnar y Tibi, que por su mayor dinamismo socioeconómico tienen un crecimiento demográfico causado por ambos saldos positivos. A su vez, y en el caso de Tibi, su situación periférica próxima a la capital provincial y su vinculación con la comarca de l'Alacantí determina la creación de flujos de nuevos residentes y de retornados, gracias, en parte, a la mejora de la comunicación por carretera ${ }^{3}$.

Los 7 municipios restantes tienen valores negativos para ambos saldos, por lo que se puede concluir que estos son los municipios que siguen en una situación más comprometida desde el punto de vista demográfico, al continuar con importantes pérdidas de población: Almudaina, Beniarrés, Benimantell, Quatretondeta, Facheca, L'Orxa y Vall de Gallinera. Muy difícil resulta establecer causas generales que expliquen el comportamiento recesivo de estos municipios, pero a las motivos relacionados con la estructura por edades con poblaciones fuertemente envejecidas, hay que sumar causas de origen socioeconómico y de falta de dinamismo, como sería el caso de Beniarrés, que a pesar de ser uno de los municipios de mayor tamaño demográfico tiene importantes pérdidas demográficas. Un factor para entender la dinámica de estos municipios es su localización aislada respecto al eje industrial del interior y al litoral turístico.

A continuación, en el mapa 3 se diferencian los municipios según su caracterización a partir de los saldos naturales y migratorios, distinguiendo entre:

- Municipios progresivos (con ambos saldos positivos)

- Municipios con saldo natural negativo y saldo migratorio positivo

- Municipios con saldo natural positivo y migratorio negativo

- Municipios muy regresivos (con ambos saldos negativos).

3 La construcción de la Autovía Central, que une Alicante y Valencia por el interior, ha sido clave para una mayor accesibilidad de Tibi con la capital provincial. 
La importancia de los saldos migratorios queda reflejada por el gran número de municipios que tienen saldos migratorios positivos:

- Únicamente dos municipios pueden considerarse como progresivos, al tener tanto los saldos naturales como migratorios positivos: Alqueria d'Asnar y Tibi.

- 31 municipios tienen saldos migratorios positivos frente a saldos naturales negativos.

- Alfafara y Tollos son los únicos que tienen saldos naturales positivos frente a migratorios negativos.

- 7 municipios se consideran como muy regresivos, pues tienen ambos saldos negativos, por lo que en principio serían los que se encuentran en una peor situación demográfica.

Destacan especialmente, en relación con los saldos migratorios, los flujos de nuevos residentes desde el ámbito urbano al medio rural. Estos responden a una causa principal, como es la revalorización del medio rural como lugar de residencia por las potencialidades que ofrece como medio habitable frente al medio urbano. En nuestro caso, destacan los flujos originados desde las cabeceras comarcales hacia los pequeños municipios rurales, por las distancias relativamente cortas que hay que recorrer hasta las mismas y donde se concentran los servicios básicos para la población.

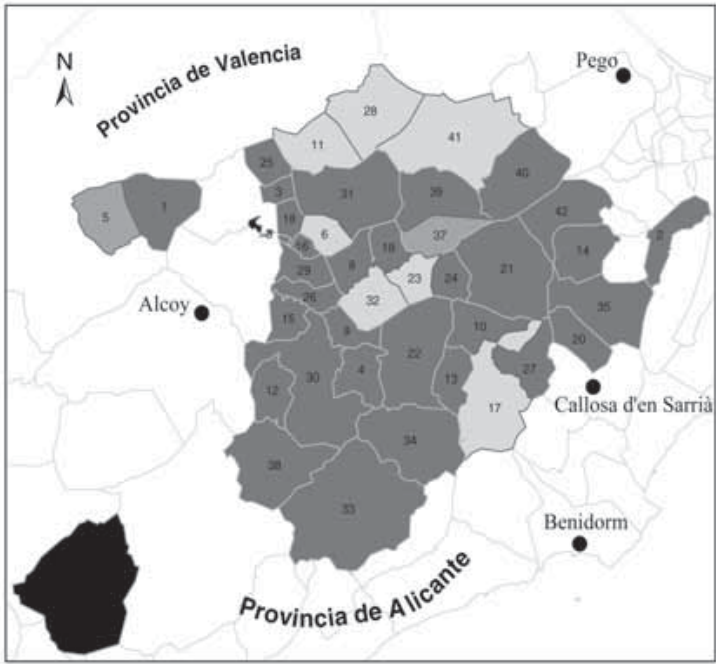

Fuente: INE e IVE. Censos de población y Anuaris estadistics de la Comunitat Valenciana
Municipios de la "Montaña de Alicante"

\begin{tabular}{|l|l|}
\hline 1. Agres & 22. Confrides \\
\hline 2. Alealali & 23. Facheca \\
\hline 3. Alcoecer de Planes & 24. Famorea \\
\hline 4. Alcolecha & 25. Gayanes \\
\hline 5. Alfafara & 26. Gorga \\
\hline 6.Almudaina & 27. Guadalest \\
\hline 7. Alqueria d'Asnar & 28. L'Orxa \\
\hline 8. Balones & 29. Millena \\
\hline 9. Benasau & 30. Penáguila \\
\hline 10.Beniarda & 31. Planes \\
\hline 11. Beniarrés & 32. Quatretondeta \\
\hline 12. Benifallim & 33. Relleu \\
\hline 13. Benifato & 34. Sella \\
\hline 14. Benichembla & 35. Tárbena \\
\hline 15. Benilloba & 36. Tibi \\
\hline 16. Benillup & 37. Tollos \\
\hline 17. Benimantell & 38. Torremanzanas \\
\hline 18. Benimantell & 39. Vall.d'Alcala \\
\hline 19. Benimassot & 40. Vall de Ebo \\
\hline 20. Bolulla & 41. Vall de Gallinera \\
\hline 21. Castell de Castells & 42. Vall de Laguart \\
\hline
\end{tabular}

Tendencias demográficas en los municipio rurales de la "Montaña de Alicante"

Municipios progresivos (saldos naturales y migratorios positivos) Municipios con saldo natural negativo y saldo migratorio positivo Municipios con saldo natural positive y saldo migratorio negativo Municipios regresivos (saldos naturales y megratorios negativos)

MAPA 3. Tendencias demográficas (a partir de los saldos naturales y migratorios) en los municipios rurales de la «Montaña de Alicante». Periodo 1991-2001. 


\section{La llegada de población extranjera}

A los flujos internos hay que sumar los originados por la llegada de población extranjera, sobre todo de ciudadanos comunitarios que establecen su residencia en relación con un turismo residencial en expansión. Sin olvidar los más recientes procesos de llegada de inmigrantes extracomunitarios por causas socioeconómicas, aunque, por ahora, se trata de flujos muy reducidos en relación con la atracción y las posibilidades de trabajo que se dan en las cabeceras comarcales.

En algunos municipios la llegada de extranjeros es muy significativa, con porcentajes sobre la población total realmente espectaculares, como es el caso de Alcalalí (34,2 \% de extranjeros), Benigembla (32\%), Bolulla (30,7\%) y Tàrbena (22,5\%), correspondiendo en su mayoría a ciudadanos comunitarios. Esta situación se da en los municipios rurales de las comarcas de la Marina Alta y la Marina Baixa, donde la influencia de la actividad turística del litoral ha sido fundamental para entender el establecimiento de esa población. Frente a estos elevados porcentajes, más modestos resultan los tenidos en el resto de municipios de la «Montaña de Alicante», pero denotan el creciente atractivo que supone el interior montañoso frente al masificado sector litoral.

La evolución de la población extranjera durante la última década es significativa respecto a la llegada de nueva población, aunque se concentren en unos pocos municipios (ver tabla 6).

Según se indica en el cuadro, entre 1991 y 2001 la población extranjera casi se ha doblado en dicho intercensal, aunque son seis los municipios que concentran los mayores porcentajes. De este modo, entre Alcalalí, Benigembla, Bolulla, Relleu, Tàrbena y Sella, en cada una de las fechas sumaban 489 y 871 extranjeros respectivamente. El análisis de los datos de la población extranjera que reside en los municipios rurales de la «Montaña de Alicante», determina que puedan establecerse diferencias entre los casos analizados. En este sentido la diferencia estriba, principalmente, en que los ciudadanos con nacionalidad extranjera se concentran en pocos municipios, situados en el sector más oriental del ámbito de estudio, coincidiendo con el transpaís que recibe la influencia de la actividad turística del litoral marítimo: son los municipios de Alcalalí, Tàrbena, Bolulla y Benigembla, Relleu y Sella que forman una zona «fronteriza» entre «La Montaña de Alicante» y la zona litoral. Estos seis municipios concentran el $60 \%$ del total de la población extranjera considerada, cuando únicamente representan el $20 \%$ de la población total de los 42 municipios. Es decir, puede concluirse que gran parte de la población extranjera se concentra en esta zona de «borde» entre el ámbito montano y la zona litoral. Otro dato de gran interés es conocer la nacionalidad de esa población. Si se realiza una división según el origen comunitario o extracomunitario de los inmigrados, destaca que del total de extranjeros, en esos seis municipios, el 76\% de los mismos pertenecen a cuatro países de la Unión Europea.

Por otro lado, otro grupo de municipios ven reducido su número de inmigrantes extranjeros entre 1991 y 2001; destacan en este comportamiento Alcoleja, Benilloba, Benimarfull, Gorga, L'Orxa, Torremanzanas, Vall de Ebo y Vall de Laguart. Suponen en muchos casos variaciones absolutas mínimas, pero denotan que en ciertos municipios el atractivo no es el mismo, aunque las variaciones de residencia también pueden deberse a estancias temporales en nuestro país.

En el mapa 4 se puede observar la variación de población extranjera entre 1991 y 2001. Destacable es la concentración de población extranjera en el sector oriental del ámbito geográfico de estudio, debido a la influencia del turismo y de la compra de viviendas por parte de ciudadanos comunitarios. 
Tabla 6

PORCENTAJES DE POBLACIÓN EXTRANJERA RESPECTO DEL TOTAL DE POBLACIÓN MUNICIPAL. AÑOS 1991-2001

\begin{tabular}{|c|c|c|c|c|}
\hline Municipios & Extranjeros 1991 & $\begin{array}{l}\% \text { pob extrj } 1991 \\
\text { (respecto del total) }\end{array}$ & Extranjeros 2001 & $\begin{array}{l}\% \text { pob extraj } 2001 \\
\text { (respecto del total) }\end{array}$ \\
\hline Agres & 2 & 0.29 & 10 & 1,57 \\
\hline Alcalalí & 248 & 29.98 & 309 & 34,26 \\
\hline Alcocer de Planes & 5 & 4.06 & 9 & 5,88 \\
\hline Alcolecha & 8 & 2.69 & 5 & 1,99 \\
\hline Alfafara & 3 & 0.75 & 12 & 2,87 \\
\hline Almudaina & 0 & 0.0 & 3 & 2,75 \\
\hline L'Alqueria d'Asnar & 3 & 0.73 & 26 & 6,24 \\
\hline Balones & 1 & 0.65 & 20 & 10,81 \\
\hline Benasau & 1 & 0.45 & 4 & $\mathbf{1 , 8 1}$ \\
\hline Beniardà & 11 & 4.76 & 37 & 15,10 \\
\hline Beniarrés & 22 & 1.46 & 21 & 1,53 \\
\hline Benigembla & 48 & 13.55 & 161 & 32,99 \\
\hline Benifallim & 0 & 0.0 & 0 & 0 \\
\hline Benifato & 9 & 7.25 & 24 & 14,46 \\
\hline Benilloba & 19 & 1.98 & 6 & 0,66 \\
\hline Benillup & 2 & 2.63 & 2 & 2,06 \\
\hline Benimantell & 17 & 3.72 & 49 & 12,19 \\
\hline Benimarfull & 12 & 2.88 & 6 & 1,43 \\
\hline Benimasot & 5 & 4.23 & 3 & 2,04 \\
\hline Bolulla & 47 & 16.9 & 110 & 30,73 \\
\hline Castell de Castells & 14 & 2.63 & 18 & $\mathbf{3 , 8 2}$ \\
\hline Confrides & 9 & 3.03 & 22 & 7,12 \\
\hline Cuatretondeta & 9 & 4.5 & 13 & 7,83 \\
\hline Facheca & 2 & 1.45 & 1 & $\mathbf{0 , 8 7}$ \\
\hline Famorca & 7 & 10.0 & 13 & 17,81 \\
\hline Gaianes & 5 & 1.51 & 2 & 0.63 \\
\hline Gorga & 6 & 2.06 & 2 & 0,74 \\
\hline Guadalest & 7 & 4.24 & 19 & 10,56 \\
\hline L'Orxa & 16 & 1.97 & 7 & 0,95 \\
\hline Millena & 2 & 1.48 & 14 & 7,87 \\
\hline Penàguila & 0 & 0.0 & 7 & 1,96 \\
\hline Planes & 21 & 2.7 & 25 & 3,18 \\
\hline Relleu & 11 & 1.48 & 87 & 10,88 \\
\hline Sella & 25 & 4.23 & 45 & 7,61 \\
\hline Tárbena & 63 & 8.83 & 159 & 22,24 \\
\hline Tibi & 21 & 2.00 & 51 & 4,07 \\
\hline Tollos & 3 & 7.14 & 6 & 14,62 \\
\hline Torremanzanas & 32 & 4.34 & 28 & 3,98 \\
\hline Vall d'Alcalà & 10 & 5.74 & 9 & 5,42 \\
\hline Vall de Ebo & 17 & 4.73 & 11 & 3,46 \\
\hline Vall de Gallinera & 24 & 3.26 & 46 & $\begin{array}{l}, 7,32 \\
7,32\end{array}$ \\
\hline Vall de Laguart & 42 & 4.25 & 31 & 3,56 \\
\hline Total & 809 & 4.46 & 1.433 & 7,92 \\
\hline
\end{tabular}

Fuente: INE. Censos de Población de 1991 y 2001. Elaboración propia. 

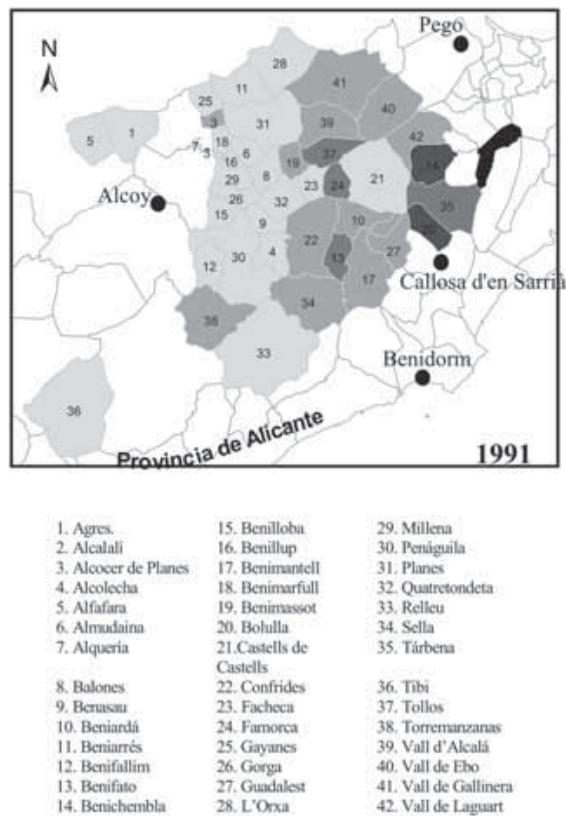

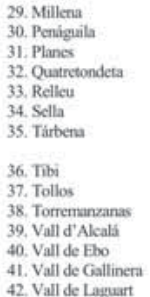

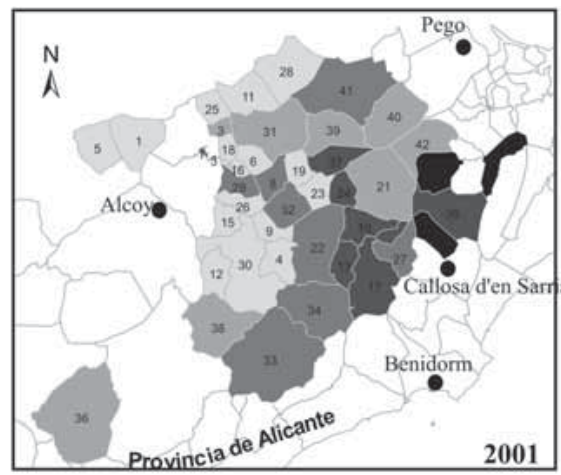

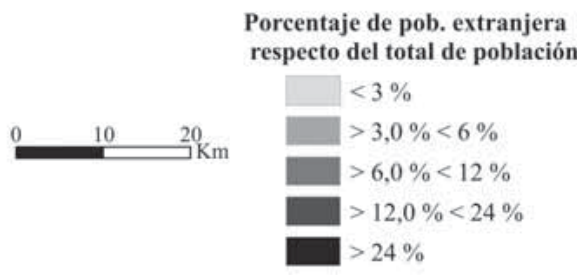

Fuente: Censos de Población 1991 y 2001. Elaboración propia

MAPA 4. Comparación de los porcentajes de población extranjera. Años 1991 y 2001. Municipios rurales de menos de 2.000 habitantes. Ámbito geográfico «Montaña de Alicante».

\section{Las consecuencias de una estructura de población fuertemente envejecida}

El envejecimiento se ha constituido en una variable trascendente en los países desarrollados, por sus consecuencias de carácter económico y social (ABELLÁN et al, 1989: 361), y más concretamente en el medio rural, ya que en éste se alcanzan las tasas de envejecimiento más acusadas, estableciéndose entonces una implicación directa entre envejecimiento y grado de ruralidad (GARCÍA et al, 1998: 274).

En este estudio se utilizará una metodología para clasificar a los municipios rurales según el mayor o menor grado de envejecimiento, lo que permite un análisis diferenciado. Esta es la propuesta utilizada por P. Paillat y A. Parant ${ }^{4}(1990)$; se basa en diferenciar tres grandes grupos según las tasas de envejecimiento al considerar la población mayor de 65 años. De este modo, según la metodología utilizada por estos autores, se considera que porcentajes de envejecimiento inferiores a un $15 \%$ corresponden a municipio rurales «jóvenes», del 16 al $19 \%$ serían municipios «en transición», entre un 20 y un $23 \%$ corresponde a municipios rurales «viejos», y, por último si se supera el $24 \%$ de población mayor de 65 años se puede hablar de municipios rurales «muy viejos» 5 .

4 P. Paillant et A. Parant, I., Le vielliesment de la campagne française, Paris, INED, Travaux et Documents, Cahier no 88, 1990, 294 pp.

5 Creemos oportuno introducir una pequeña matización a la hora de utilizar esta metodología, ya que con los umbrales que se establecen no se llega a apreciar de forma idónea la realidad del problema del envejecimiento en el área estudiada. Se ha establecido la variación siguiente: el intervalo de porcentajes para los municipios «viejos» comprenderá, a partir de ahora, del 20 al 34\%, mientras que para los municipios «muy viejos», este pasará a considerarse a partir de valores iguales o superiores al $35 \%$. 
A partir de los umbrales utilizados por los autores anteriormente mencionados, se modificarán los intervalos propuestos para adaptarse mejor a la realidad de los municipios de «La Montaña de Alicante». Por este motivo se establecen las siguientes modificaciones (ver mapas):

- Para el intervalo de los municipios viejos se considerará un rango que abarque desde porcentajes de envejecimiento del 20 al 34\%, frente al propuesto que comprendía un intervalo reducido entre el 20 y $23 \%$

- El intervalo de municipios «muy viejos» comprenderá los porcentajes que superen el $35 \%$, cuando en la propuesta tomada como referencia este intervalo se consideraba a partir del porcentaje superior a un $24 \%$

A continuación se muestra en la tabla 7 la evolución de las tasas de envejecimiento para los diferentes municipios del ámbito considerado, en el que se toma como referencia el periodo de tiempo comprendido entre 1981 y 2001, periodo que se caracteriza por un proceso de envejecimiento muy acusado.

Las causas de las altas tasas de envejecimiento en la comarca son las siguientes:

- El proceso de éxodo rural ocurrido durante todo el siglo XX ha influido para que en los municipios objeto de estudio se produjese un fuerte éxodo rural de la población más joven, quedando paulatinamente los grupos de población más envejecidos. La población emigraba por el atractivo que suponían las posibilidades económicas que ofrecían las cabeceras comarcales o las comarcas vecinas, como ocurrió, por ejemplo, hacia el eje urbano industrial de Alcoy-Cocentaina- Muro (proceso iniciado en Alcoy y que posteriormente se difundió por el resto de los municipios). El factor inicial que mayor repercusión tuvo en el medio rural fue el paso desde un proceso productivo descentralizado, en el cual muchas fases productivas eran realizadas por el campesinado ${ }^{6}$ en municipios cercanos, al modelo fabril centralizado en las cabeceras comarcales. De este modo, dos son los factores que explican el despoblamiento: la crisis agraria y el desarrollo industrial en las ciudades. Procesos que se iniciaron en el siglo XIX, pero que continuaron durante la primera mitad del s. XX y al que sucedieron nuevas pérdidas demográficas, sobre todo a partir de 1950 y hasta bien entrada la década de los ochenta, fruto de las oportunidades que se crearon en las cabeceras comarcales y por la escasez de alternativas en el medio rural. Por otro lado, no debe olvidarse que existen otros municipios que quedan fuera de esta influencia, como son los municipios de las Marinas, en los que la atracción ejercida por el desarrollo económico del litoral de la provincia de Alicante fue mucho más tardía y responde a otra realidad totalmente distinta a la expuesta anteriormente. En definitiva todos estos procesos redundaron en un fuerte despoblamiento hacia áreas cercanas pero mucho más dinámicas

- Las poblaciones cada vez más envejecidas han ido reduciendo sus tasas de natalidad por el importante descenso de los grupos de población en edad de procrear, especialmente desde los años cincuenta del siglo XX. Este fenómeno se acentuó, más si cabe por la consolidación de los medios urbanos ante la falta de expectativas que se estaban dando en el medio rural por la paulatina desagrarización y por la creciente falta de oportunidades.

6 También conocido como «Putting out system». 
Tabla 7

EVOLUCIÓN DE LAS TASAS DE ENVEJECIMIENTO DURANTE LAS DOS ÚLTIMAS DÉCADAS EN LOS MUNICIPIOS RURALES DE LA «MONTAÑA DE ALICANTE»

\begin{tabular}{|c|c|c|c|}
\hline Municipios & T. evj 1981 & T. evj 1991 & T. evj 2001 \\
\hline Agres & 18,71 & 24,11 & 25,04 \\
\hline Alcalalí & 17,15 & 26,60 & 31,04 \\
\hline Alcocer & 18,94 & 32,52 & 26,14 \\
\hline Alcoleja & 28,03 & 28,96 & 33,07 \\
\hline Alfafara & 19,06 & 18,80 & 20,57 \\
\hline Almudaina & 43,83 & 31,58 & 31,19 \\
\hline L'Alqueria d'Asnar & 11,17 & 21,32 & 26,38 \\
\hline Balones & 24,02 & 25,66 & 28,11 \\
\hline Benasau & 24,80 & 26,70 & 27,15 \\
\hline Beniardà & 31,05 & 31,17 & 28,16 \\
\hline Beniarrés & 17,20 & 18,90 & 27,35 \\
\hline Benigembla & 20,54 & 33,05 & 31,56 \\
\hline Benifallim & 22,45 & 22,89 & 32,26 \\
\hline Benifato & 25,00 & 29,03 & 22,89 \\
\hline Benilloba & 21,43 & 21,06 & 23,92 \\
\hline Benillup & 74,19 & 19,74 & 16,49 \\
\hline Benimantell & 30,35 & 24,28 & 28,86 \\
\hline Benimarfull & 24,05 & 28,37 & 30,48 \\
\hline Benimassot & 22,61 & 32,20 & 21,77 \\
\hline Bolulla & 28,43 & 30,69 & 30,45 \\
\hline Castells de Castells & 24,17 & 32,14 & 38,00 \\
\hline Confrides & 44,03 & 33,33 & 32,04 \\
\hline Cuatretondeta & 45,86 & 38,50 & 36,52 \\
\hline Facheca & 12,36 & 21,90 & 47,95 \\
\hline Famorca & 23,02 & 51,43 & 29,78 \\
\hline Gaianes & 24,58 & 28,10 & 31,23 \\
\hline Gorga & 27,55 & 20,27 & 21,67 \\
\hline Guadalest & 13,41 & 19,39 & 27,95 \\
\hline l'Orxa & 20,26 & 23,33 & 23,03 \\
\hline Millena & 14,29 & 25,93 & 28,57 \\
\hline Penàguila & 26,24 & 28,13 & 28,21 \\
\hline Planes & 20,76 & 27,83 & 42,17 \\
\hline Relleu & 22,04 & 30,32 & 29,75 \\
\hline Sella & 21,88 & 26,78 & 31,47 \\
\hline Tàrbena & 35,41 & 34,78 & 34,83 \\
\hline Tibi & 14,30 & 15,69 & 20,45 \\
\hline Tollos & 55,10 & 47,62 & 36,59 \\
\hline Torremanzanas & 15,35 & 22,28 & 28,55 \\
\hline Vall d'Alcalà & 28,89 & 41,95 & 45,18 \\
\hline Vall de Ebo & 25,29 & 36,77 & 36,79 \\
\hline Vall de Gallinera & 26,02 & 34,97 & 33,01 \\
\hline Vall de Laguart & 23,48 & 26,01 & 31,61 \\
\hline Total & 22,3 & 26,25 & 28,97 \\
\hline
\end{tabular}

Fuente: INE. Censos de la población de España. Elaboración propia. 
- Además debe de tenerse en cuenta los cambios generales en las pautas de fecundidad, ocurridos desde mediados de la década de los setenta y que contribuyeron a reducir todavía más las tasas de natalidad ${ }^{7}$.

La evolución seguida en todo el ámbito geográfico analizado ha sido la de incrementar notablemente sus tasas de envejecimiento entre 1981 y 2001. Mientras que para la primera fecha el envejecimiento afectaba al 22,3\% de la población, para el año 2001 este valor se había incrementado hasta el 29\% (ver mapa 5).

Como se observa en los mapas, las altas tasas de envejecimiento son generales en los tres periodos considerados, aunque se produce una progresiva generalización de los municipios considerados como «viejos» desde 1981 a 2001, siendo también significativa la localización de los municipios «muy viejos» (tonos más oscuros) que espacialmente se sitúan en el área central del ámbito geográfico estudiado. El problema del envejecimiento es muy importante por su significado económico, al tratarse de la población que deja el
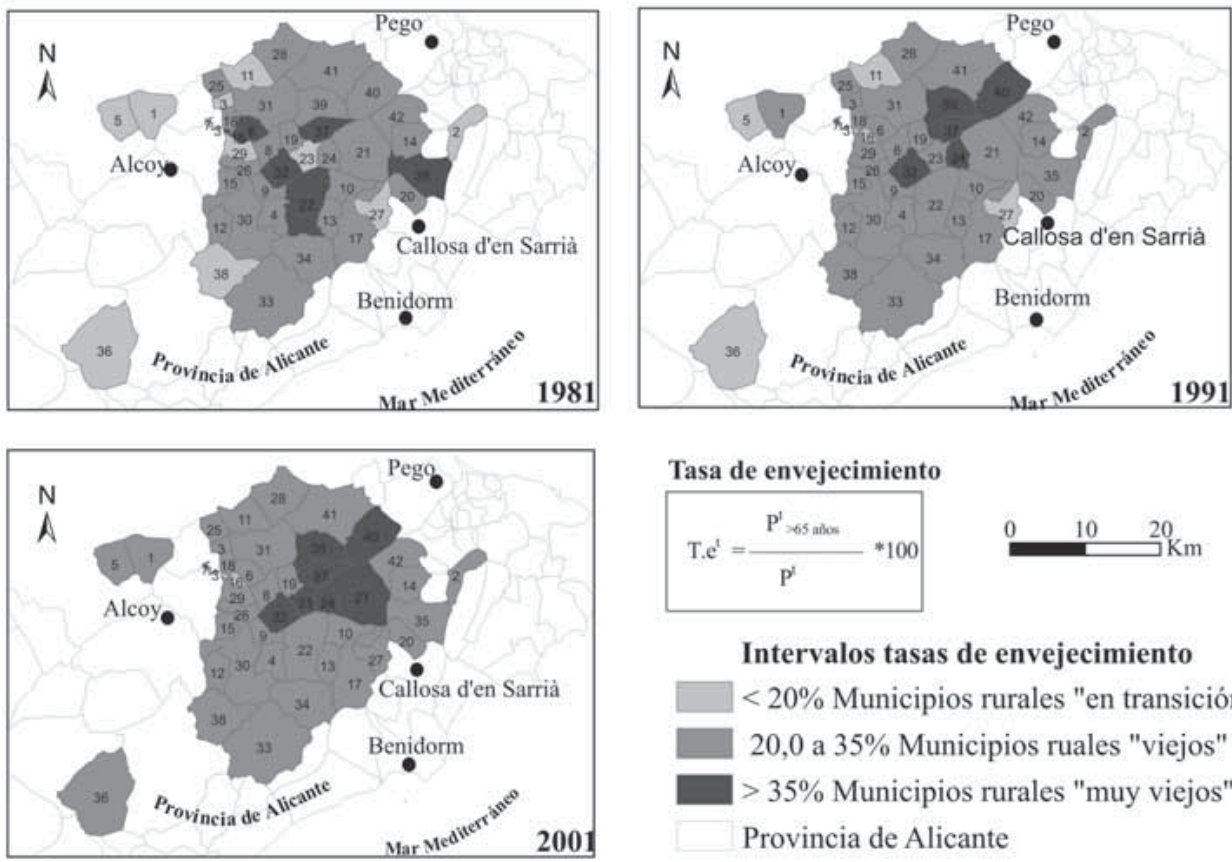

Tasa de envejecimiento

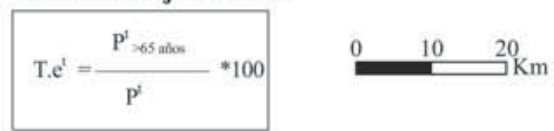

\section{Intervalos tasas de envejecimiento \\ $<20 \%$ Municipios rurales "en transición" \\ 20,0 a $35 \%$ Municipios ruales "viejos" \\ $>35 \%$ Municipios rurales "muy viejos" \\ Provincia de Alicante}

Fuente: INE, Censos de Población 1981,1991 y 2001 Elaboración propia

MAPA 5. Evolución de las Tasas de envejecimiento para los años censales 1981, 1991 y 2001. Municipios rurales de la «Montaña de Alicante».

7 Proceso general para el conjunto de España donde a partir de 1976 se producen descensos considerables de las tasas de natalidad y fecundidad. Progresivamente, se fueron reduciendo dichos índices hasta alcanzar mínimos desde la década de los noventa. 
periodo laboral, además del deterioro físico progresivo que lleva a la necesidad de incrementar determinados servicios sanitarios y asistenciales (GOZÁLVEZ, 1987: 60).

\section{Conclusiones}

Tras analizar la situación demográfica actual de los municipios de la «Montaña de Alicante», se establecen las siguientes conclusiones:

\section{a) Inicio de un cambio de tendencia demográfica}

Del éxodo rural generalizado, se ha pasado incluso a la recuperación demográfica, aunque, todavía hoy, se producen pérdidas demográficas en algunos municipios, que responden, por su cuantía, más bien a un saldo natural negativo y no a un éxodo rural propiamente dicho. En cifras, el cambio de tendencia se aprecia por la evolución de los porcentajes de variación de la población entre los periodos 1981-1991 y 1991-2001, de este modo si en el primer decenio el descenso de población alcanzaba un valor de $-10,38 \%$, durante la última década éste se ha reducido a tan sólo un $-0,20 \%$.

La estructura demográfica fuertemente envejecida ha generado una dinámica poco favorable a la recuperación demográfica por efecto de los saldos naturales, debido a la alta mortalidad - como consecuencia del gran número de personas en edad avanzada - y a la escasez de nacimientos por la reducción de los grupos en edad de procrear. A pesar de esta situación, la recuperación demográfica viene dada, sobre todo, por los saldos migratorios positivos, que llegan a contrarrestar las pérdidas originadas por los saldos naturales.

b) Una doble dinámica demográfica en los municipios rurales de la «Montaña de Alicante»: diferencias de comportamiento

Puede hablarse de una doble dinámica demográfica entre los municipios rurales de la «Montaña de Alicante», por una parte hablaríamos de los municipios con una continuada regresión demográfica durante las dos últimas décadas y, por otra, la de aquellos municipios que crecen demográficamente. Estas significativas diferencias entre municipios, responden a la propia dinámica socioeconómica de los mismos, mucho más favorable a la recuperación en los municipios situados en el sector este del ámbito de estudio, en el interior de las comarcas de la Marina Alta y la Marina Baixa.

Entre los municipios con un mayor dinamismo demográfico durante los años noventa destacan: Benigembla (con crecimiento del 37,85\% entre 1991 y 2001), Benifato $(33,87 \%)$, Millena (31,83\%), Bolulla (29,24\%), Benillup (27,63\%), Benimassot (24,58\%), Alcocer de Planes (24,39\%), Balones (21,71\%), Tibi (19,81\%), Alcalalí $(9,07 \%)$ y Relleu (7,82\%). Por el contrario, los que pierden más población entre 1991 y 2001 son: Almudaina $(-18,05 \%)$, Quatretondeta (-17\%), Facheca (-16,06\%), Alcoleja (-15,49\%), Vall de Gallinera $(-15,1 \%)$, Vall de Laguart $(-11,94 \%)$, Castells de Castells $(-11,47 \%)$, Vall d'Ebo $(-11,42 \%)$, Benimantell $(-11,2 \%)$ y Beniarrés $(-8,5 \%)$.

\section{c) Los nuevos residentes en el medio rural}

Los saldos migratorios positivos son fundamentales para conocer la realidad de los municipios rurales objeto de estudio. La llegada de nueva población ha generado, sin duda, nuevas dinámicas socioeconómicas que rompen con la situación de decadencia continua que ha sufrido el medio rural del interior norte de la provincia de Alicante. Con 
todo, esta situación no puede generalizarse para todo el ámbito geográfico considerado, porque, como ya se ha comentado anteriormente, aún hoy, existen procesos demográficos recesivos que definen la complicada situación debida a la propia estructura demográfica de la población.

Destacan especialmente, en relación con la llegada de nueva población, los flujos de nuevos residentes desde el ámbito urbano al medio rural. Los así originados responden a una causa principal, como es la revalorización del medio rural como lugar de residencia por las potencialidades que ofrece como medio habitable frente al medio urbano. En nuestro caso, destacan los flujos originados desde las cabeceras comarcales hacia los pequeños municipios rurales, por las relativas cortas distancias que hay que recorrer hasta las mismas y donde se concentran los servicios básicos para la población.

\section{d) La población extranjera en el medio rural: nuevos procesos demográficos}

A los flujos anteriores hay que sumar los originados por la llegada de población extranjera, sobre todo de ciudadanos comunitarios que establecen su residencia en relación con un turismo residencial en expansión. Sin olvidar los más recientes procesos de llegada de inmigrantes extracomunitarios por causas socioeconómicas, aunque por ahora se trata de flujos muy reducidos y en relación con la atracción y las posibilidades de trabajo que se dan en las cabeceras comarcales.

En algunos municipios la llegada de extranjeros es muy significativa, con porcentajes sobre la población total realmente espectaculares, como es el caso de Alcalalí $(34,2 \%$ de extranjeros), Benigembla (32\%), Bolulla $(30,7 \%)$ y Tàrbena $(22,5 \%)$, correspondiendo en su mayoría a ciudadanos comunitarios. Esta situación se da en los municipios de las comarcas de la Marina Alta y la Marina Baixa, donde la influencia de la actividad turística del litoral ha sido fundamental para entender el establecimiento de esa población. Frente a estos elevados porcentajes, más modestos resultan en el resto de municipios de la montaña de Alicante, pero denotan el atractivo que supone el interior montañoso frente al masificado sector litoral

\section{e) El envejecimiento de la población: un problema común con graves consecuencias para el futuro}

La característica común a todos los municipios es la de tener unas elevadas tasas de envejecimiento, con las consecuencias futuras que ello puede tener para los municipios rurales, ya que la estructura demográfica determina, por ejemplo, que los saldos naturales en un futuro continúen siendo negativos. Situación que realmente no cambiará si no se produce una regeneración demográfica por rejuvenecimiento de la población. Situación que, para ser posible, debería producirse por la llegada de población joven, que a su vez podría incrementar los nacimientos y compensar de este modo las elevadas tasas de mortalidad. El elevado grado de envejecimiento determina tasas de dependencia muy elevadas que denotan el fuerte desequilibrio entre una población envejecida y la escasa proporción de los grupos de población en edad activa, situación complicada desde el punto de vista social por la dificultad de garantizar el cuidado de las personas de mayor edad, así como del desarrollo socioeconómico de los municipios.

Por otro lado, la situación socioeconómica por la que pasan estos municipios es determinante para comprender las pautas que se seguirán en un futuro. En este sentido, al igual que en la cuestión demográfica, parece que el tradicional estancamiento socioeconómico, se intenta superar con muevas iniciativas para dinamizar la economía del medio rural. Las recientes 
iniciativas en relación con el desarrollo rural, sin duda, son fundamentales para vertebrar territorialmente este espacio y permiten la creación de nuevas potencialidades que deben de ayudar a la mejora demográfica de los municipios rurales de la «Montaña de Alicante».

\section{f) La amenaza de la especulación inmobiliaria}

Uno de los problemas a los que se enfrentan los pequeños municipios rurales de la «Montaña de Alicante» es el de la especulación inmobiliaria. La influencia de determinados modelos desarrollados en el litoral, en el que priman la creación de grandes proyectos inmobiliarios, ya se ha trasladado al interior montañoso de la provincia de Alicante. Por citar sólo dos ejemplos, en Guadalest y Sella se han proyectado la construcción de dos grandes urbanizaciones que, para el primer caso pretendían acoger a más de 1.000 nuevos habitantes, cuando el municipio solo cuenta con 300. Proyectos de este tipo son los que deben ser rechazados por sus negativas consecuencias sociales, medioambientales y culturales. El peligro de desvirtuar la cultura y el patrimonio, así como las consecuencias desde el punto de vista social, en cuanto a la repercusión demográfica y en relación a la dotación de servicios básicos para la población, hace de estas propuestas el principal factor a tener en cuenta durante el futuro más próximo.

Desde el punto de vista demográfico, esta situación implicaría el desmesurado crecimiento de la población, con la problemática añadida de la falta de servicios que caracteriza al medio rural. A su vez, si la nueva población responde a un tipo de demanda en relación con el turismo residencial que, mayoritariamente, se compone de personas en edad avanzada, el problema del envejecimiento sería todavía mayor, con las repercusiones que ello supondría en cuanto a la dinámica socioeconómica.

En definitiva, el desarrollo de los municipios rurales debe basarse en la puesta en valor de lo propio, permitiendo y favoreciendo la llegada «racional» de nueva población y, todo ello, en relación a los nuevos procesos generados a partir de la revalorización del medio rural.

\section{Bibliografía}

ABELLÁN GARCÍA, A.; RODRÍGUEZ, V. (1989): «Proceso de envejecimiento de la población española 1970-1981)» en Análisis del desarrollo de la Población Española en el periodo 19701986. Ed. Síntesis. Grupo de Población de la AGE. Madrid, pp. 357-364.

GARCÍA, A.; PUGA, D.; SÁNCHEZ, D. (1998): «Envejecimiento demográfico en el País Valenciano: contrastes territoriales e implicaciones socio-demográficas» en Actas de las II Jornadas de Estudios sobre la Población Valenciana, La población valenciana, pasado, presente y futuro, Vol. 2. Instituto de Cultura Juan Gil-Albert, Alicante, pp. 257-281.

GOZÁLVEZ PÉREZ, V. (1972): «Notas sobre demografía de la provincia de Alicante», Cuadernos de Geografía, no 11, Universidad de Valencia, pp. 27-77.

GOZÁLVEZ PÉREZ, V. (1987): «El envejecimiento de la población en los municipios rurales del País Valenciano», Cuadernos de Geografía, no 41, Universidad de Valencia, pp. 59-74.

MATARREDONA COLL, E. (1996): «Atonía y agotamiento demográfico de la Montaña Alicantina», Investigaciones Geográficas, $\mathrm{n}^{\circ}$ 14, Instituto de Geografía de la Universidad de Alicante, pp. 41-60.

PAILLANT, P.; PARANT, A., (1980): Le vieillissement de la campagne française. Paris, INED, Travaux et Documents, Cahier n ${ }^{\circ} 88,1990,294$ pp.

PÉREZ i PÉREZ, D. (1997): Reestructuració del espais industrials de l'eix Alcoi-Cocentaina-Muro. Ed. Instituto de Cultura Juan Gil Albert, Alicante, 449 páginas.

VINUESA, J. (editor) y otros. (1997): Demografía, análisis y proyecciones, Ed. Síntesis, Madrid, 366 páginas. 
